\title{
LA REPITENCIA EN LA CULMINACIÓN DEL PENSUM DE ESTUDIOS DE LAS CARRERAS DE PREGRADO DE LA UNIVERSIDAD UTE
}

\section{REPETITION IN THE COMPLETION OF THE CURRICULUM OF STUDIES IN THE UNDERGRADUATE PROGRAMS OF UTE UNIVERSITY}

Juan Carlos Rivera Gaiborl, Gabriel Enrique Pazmiño Castillo², Nelson Alberto Cerda Prado3

\section{Resumen}

El estudio tiene como objetivo analizar el tiempo requerido para aprobar la malla curricular (pensum de estudios) considerando el impacto que tiene la repitencia en el porcentaje de avance. La población a analizar es la de los estudiantes matriculados en el periodo académico abril 2017 / agosto 2017 de la Sede Quito. Los datos provienen del Sistema Integrado de Control Académico y Financiero (SICAF) de la Universidad Tecnológica Equinoccial (UTE). Para el análisis de los datos se utilizó la hoja electrónica Excel y Microsoft Access. De los datos se obtiene que el número máximo de periodos requeridos para culminar para aprobar la malla curricular es dos, es decir, los estudiantes en promedio estarian incrementando la duración de la carrera en un 22.22\%.

\section{Palabras claves}

Rezago; repitencia

Clasificación JEL: I21J20

\section{Abstract}

The study aims to analyze the time required to approve the curriculum (program of study) considering the impact that repetition has on the percentage of progress. The population to be analyzed is that of the students enrolled in the semester April 2017 - August 2017 of the Quito Campus ( $\cap$ = 10454). The data comes from the Integrated System of Academic and Financial Control "SICAF" of the UTE University. For the data analysis we used Excel Electronic Sheet and Microsoft Access. From the data, the results established that the maximum number of additional semesters required to complete and approve the curriculum is two; which means that students would be increasing the duration of their undergraduate studies in an average of $22.22 \%$

\section{Keywords}

Lag, repetition

JEL Classification: $121 \mathrm{~J} 20$ 


\section{Metodología}

En el artículo se realiza una revisión de la literatura sobre la repitencia de las carreras de pregrado. Se analiza la estructura de cada pensum de estudios (cantidad de asignaturas, número de créditos y niveles) de las seis facultades de la UTE. Del SICAF, se extrajeron los datos de la situación de los estudiantes matriculados en cada una de las carreras a la finalización del periodo académico abril / agosto 2017, con el uso del programa Microsoft Access se obtuvieron los datos auxiliares y agregados por facultad como carrera, género, número de matrícula, cantidad de asignaturas aprobadas y reprobadas, porcentaje de avance, entre otros; y para la generación de cuadros se empleó la herramienta de tablas dinámicas de Microsoft Excel.

\section{Introducción}

Desde el inicio de la era industrial, las sociedades buscan aumentar la cantidad de productos obtenidos por unidad de trabajo invertido, tratando de que el incremento de la producción no reduzca la calidad, planteándose así la noción de eficiencia. Su aplicación al campo de la educación superior es directa: la principal función de una institución de educación superior (IES) es la docencia y, por tanto, su eficiencia depende principalmente de la proporción de alumnos que logran cumplir con la malla curricular y titularse, respecto a aquellos que ingresaron (López, Albíter y Ramírez, 2008).

Las causas del rezago o retraso en la culminación de la malla curricular están asociadas a la secuencia de asignaturas obligatorias establecidas por los siguientes prerrequisitos y correquisitos: el número de oportunidades para cursar una misma materia; el número permitido de materias reprobadas; los tipos de exámenes; el número de ocasiones en que puede presentarse a rendir un examen; las modalidades de titulación, y los plazos reglamentarios para concluir los estudios (Vera, Ramos, Sotelo, Echeverría y Serrano, 2012).

Guadalupe (2005) afirma que los alumnos atribuyen las problemáticas de reprobación, rezago o deserción, a la falta de orientación vocacional en el nivel medio superior, así como a la falta de promoción y difusión, específicamente de la licenciatura en la que se inscribieron. Lo anterior se debe a que el programa educativo al que ingresaron no cumplía con las expectativas trazadas al inicio (Vera et al., 2012).

La extensión de tiempo para aprobar la malla curricular afecta a las oportunidades de inserción laboral del estudiante. Esto representa un incremento en el gasto individual, familiar y de la sociedad, además afecta a la eficiencia y eficacia de la institución de educación superior.

\section{Repitencia en la culminación del pensum de estudios}

Antes de procesar la información fue necesario prepararla agregando campos adicionales como el género y el estado final para efectos de tener únicamente tres estados: aprobado, reprobado y retirado. También fue necesario normalizar el periodo a un formato de año y mes. Como se observa en la tabla 1.

También se consideraron las matrículas en materias optativas y obligatorias del periodo abril / agosto 2017, y no se tomaron en cuenta las materias retiradas. 
Tabla 1. Muestra la información de la base de datos proporcionada para cada materia del estudiante y una descripción de cada campo

\begin{tabular}{|c|c|}
\hline Campo & Descripción \\
\hline Facultad & Nombre de la facultad en la que está matriculado el estudiante \\
\hline Escuela & Nombre de la escuela en la que está matriculado el estudiante \\
\hline Cedula & Número de la cédula del estudiante \\
\hline Nombres & Apellidos y nombres del estudiante \\
\hline Materia & Materia del estudiante \\
\hline Nivel & Nivel que corresponde a la materia \\
\hline N. ${ }^{0}$ de créditos & Número de créditos del estudiante \\
\hline Periodo & $\begin{array}{l}\text { Es el periodo en el cual tomó la materia, si la materia no ha sido tomada por el } \\
\text { estudiante aparece en blanco }\end{array}$ \\
\hline Estado de la materia & $\begin{array}{l}\text { Tiene varias opciones e indica el estado de la materia al final del periodo, estas } \\
\text { opciones son para: } \\
\text { Aprobado: } \\
\text { "APROBADO" } \\
\text { "EXONERADO" } \\
\text { "EXAMEN UBICACIÓN" } \\
\text { "HOMOLOGADO" } \\
\text { Reprobado: } \\
\text { "REPROBADO" } \\
\text { "REPRUEBA ASIS" } \\
\text { "FINAL" } \\
\text { "REPOSICIÓN" } \\
\text { "PENDIENTE" } \\
\text { Retirado: } \\
\text { Si el estudiante retiró la matrícula consta "RETIRADO" } \\
\text { Si el estudiante no se ha matriculado en la materia consta "POR APROBAR" en } \\
\text { este campo. }\end{array}$ \\
\hline $\begin{array}{l}\text { Número de veces que tomo la } \\
\text { materia }\end{array}$ & $\begin{array}{l}\text { Indica cuantas veces ha tomado la materia el estudiante, si el valor es } 0 \text { signi- } \\
\text { fica que el estudiante no se ha matriculado en la materia }\end{array}$ \\
\hline Tipo materia & $\begin{array}{l}\text { Indica si la materia es de alguno de los siguientes tipos: } \\
\text { En blanco: } \\
\text { corresponde al curso de nivelación } \\
\text { "ANUAL": } \\
\text { para materias de pensum antiguos de carreras } \\
\text { "INDUCCIÓN": } \\
\text { corresponde a curso para modalidad a distancia } \\
\text { "LABORATORIO": corresponde a materias específicas }\end{array}$ \\
\hline
\end{tabular}

\section{Matrícula del periodo académico abril / agosto 2017}

Para efectos de analizar la repitencia en la culminación del pensum de estudios, como se menciona anteriormente, se tomaron del SICAF para el periodo académico abril / agosto 2017 los siguientes datos de los estudiantes matriculados: 
- La matrícula por asignatura y el número de matrículas, así como resultado por materia al final del periodo

- Número de asignaturas registradas

- Número de materias reprobadas

- Número de créditos registrados

- Número de créditos aprobados

- Porcentaje de avance del pensum de estudios

En el periodo señalado, en las seis facultades, se matricularon 10454 estudiantes de los cuales el 51,03\% corresponde al género femenino y el $48,97 \%$ al género masculino, sin embargo a pesar de haber un porcentaje similar de mujeres y hombres matriculados en la Universidad, se observa que en las facultades de Comunicación, Artes y Humanidades y en Ciencias de la Salud el número de mujeres supera considerablemente al de hombres y únicamente en la Facultad de Ciencias de la Ingeniería e industrias el número de hombres es significativamente mayor al de mujeres, como se detalla en la Tabla 2

Tabla 2. Número de estudiantes matriculados por género y facultad

\begin{tabular}{|l|l|l|l|l|l|}
\hline \multicolumn{1}{|c|}{ FACULTAD } & FEMENINO & MASCULINO & TOTAL & $\begin{array}{c}\text { \% } \\
\text { FEMENINO }\end{array}$ & $\begin{array}{c}\text { \%ASCULINO } \\
\text { MAN }\end{array}$ \\
\hline Arquitectura y Urbanismo & 324 & 390 & $\mathbf{7 1 4}$ & $\mathbf{4 5 , 3 8}$ & 54,62 \\
\hline Ciencias Administrativas & 1570 & 1163 & $\mathbf{2 7 3 3}$ & $\mathbf{5 7 , 4 5}$ & $\mathbf{4 2 , 5 5}$ \\
\hline Ciencias de la Ingeniería e Industrias & 608 & 1898 & $\mathbf{2 5 0 6}$ & $\mathbf{2 4 , 2 6}$ & $\mathbf{7 5 , 7 4}$ \\
\hline Ciencias de la Salud & 570 & 368 & $\mathbf{9 3 8}$ & $\mathbf{6 0 , 7 7}$ & 39,23 \\
\hline Comunicación, Artes y Humanidades & 1662 & 777 & $\mathbf{2 4 3 9}$ & $\mathbf{6 8 , 1 4}$ & 31,86 \\
\hline Hospitalidad y Servicios & 601 & 523 & $\mathbf{1 1 2 4}$ & 53,47 & $\mathbf{4 6 , 5 3}$ \\
\hline TOTAL & $\mathbf{5 3 3 5}$ & $\mathbf{5 1 1 9}$ & $\mathbf{1 0 4 5 4}$ & $\mathbf{5 1 , 0 3}$ & $\mathbf{4 8 , 9 7}$ \\
\hline
\end{tabular}

Fuente: SICAF

En cuanto a las asignaturas que tomaron los estudiantes, periodo analizado, se observa que los 10,454 estudiantes registrados en las diferentes facultades se matricularon en 57,875 asignaturas. La tabla 3 muestra el número de materias tomadas por los estudiantes según el número de matrícula; se puede observar en la mencionada tabla que del número de materias tomadas por los estudiantes el 93,61\% corresponde a primera, $5,71 \%$ a segunda y $0,68 \%$ a tercera matricula. A nivel de facultad revela que la que tiene el mayor porcentaje de segundas matrículas es Arquitectura y Urbanismo, y la que tiene el porcentaje más alto de terceras matrículas es Ciencias de la Ingeniería e Industrias.

Tabla 3. Número de materias en las que se matricularon los estudiantes por facultad según el número de matrícula

\begin{tabular}{|c|c|c|c|c|c|c|c|}
\hline Facultad & $\begin{array}{l}\text { 1RA } \\
\text { MAT. }\end{array}$ & $\begin{array}{l}\text { 2DA. } \\
\text { MAT. }\end{array}$ & $\begin{array}{l}\text { 3RA. } \\
\text { MAT. }\end{array}$ & TOTAL & $\begin{array}{c}\% \text { 1RA } \\
\text { MAT. }\end{array}$ & $\begin{array}{c}\% \text { 2DA. } \\
\text { MAT. }\end{array}$ & $\begin{array}{c}\% \text { 3RA. } \\
\text { MAT. }\end{array}$ \\
\hline Arquitectura y Urbanismo & 3925 & 311 & 34 & 4270 & 91,92 & 7,28 & 0,80 \\
\hline Ciencias Administrativas & 13492 & 762 & 107 & 14361 & 93,95 & 5,31 & 0,74 \\
\hline Ciencias de la Ingeniería e Industrias & 13143 & 986 & 126 & 14255 & 92,20 & 6,92 & 0,88 \\
\hline Ciencias de la Salud & 6793 & 123 & 1 & 6917 & 98,21 & 1,78 & 0,01 \\
\hline Comunicación, Artes y Humanidades & 11155 & 733 & 89 & 11977 & 93,14 & 6,12 & 0,74 \\
\hline Hospitalidad y Servicios & 5667 & 391 & 37 & 6095 & 92,98 & 6,42 & 0,60 \\
\hline Total & 54175 & 3306 & 394 & 57875 & $93,61 \%$ & $5,71 \%$ & $0,68 \%$ \\
\hline
\end{tabular}




\section{Resultados al final del periodo académico abril / agosto 2017}

La tabla 4 muestra, por facultad, el número y porcentaje de materias aprobadas, reprobadas y retiradas; es imperativo resaltar que el porcentaje de materias reprobadas en la Universidad sede Quito es del 7,34\%. Cabe señalar que la Facultad de Ciencias de la Salud tiene el porcentaje más bajo, 3,28\%, situación que obedece a las exigencias al personal docente para que este continuamente preparado en alcanzar mejores resultados de aprendizaje en los alumnos. De tal manera, la inversión en capacitación de los profesores debe constituirse sobre la evidencia en torno a qué tipo de cambios en la práctica del profesor tienen el mayor impacto en los logros de los estudiantes, pero también tener en cuenta cómo aprender y cómo se desarrollan los profesores (Guevara, 2016).
De las 2500 carreras de tercer nivel que se ofrecen en el país, la más solicitada fue Administración de Empresas (Cavagnaro, 2019). En el caso de la Universidad UTE la mencionada carrera se encuentra dentro de la Facultad de Ciencias Administrativas que es la más grande con respecto al total de materias.

Se puede afirmar que la tendencia de la Universidad es disminuir el porcentaje de repitencia debido a la inserción del pensamiento crítico, como reconoce Cavagnaro, (2019) que las carreras que tendrán más demanda en el futuro cercano, sin duda, son las fundamentadas en la tecnología, basadas en datos, digitales y con un gran componente de pensamiento analítico; eso no significa dejar de lado las habilidades humanas, aquellas que nos diferencian de las máquinas: empatía, pensamiento crítico y sensibilidad.

Tabla 4. Número de materias aprobadas, reprobadas y retiradas por facultad

\begin{tabular}{|c|c|c|c|c|c|c|c|}
\hline Facultad & $\begin{array}{c}\text { Número de } \\
\text { materias } \\
\text { aprobadas }\end{array}$ & $\begin{array}{c}\text { Número de } \\
\text { materias } \\
\text { reprobadas }\end{array}$ & $\begin{array}{l}\text { Nume- } \\
\text { ro de } \\
\text { materias } \\
\text { retiradas }\end{array}$ & $\begin{array}{l}\text { Total, } \\
\text { materias }\end{array}$ & $\begin{array}{c}\text { \% ma- } \\
\text { terias } \\
\text { aprobadas }\end{array}$ & $\begin{array}{c}\% \\
\text { Materias } \\
\text { reproba- } \\
\text { das }\end{array}$ & $\begin{array}{c}\% \\
\text { Materias } \\
\text { retiradas }\end{array}$ \\
\hline $\begin{array}{l}\text { Arquitectura y } \\
\text { Urbanismo }\end{array}$ & 3891 & 377 & 2 & 4270 & 91,12 & 8,83 & 0,05 \\
\hline Ciencias de la Salud & 6690 & 227 & 0 & 6917 & 96,72 & 3,28 & 0,00 \\
\hline $\begin{array}{l}\text { Comunicación, Artes y } \\
\text { Humanidades }\end{array}$ & 11086 & 879 & 12 & 11977 & 92,56 & 7,34 & 0,10 \\
\hline
\end{tabular}

ARQUITECTURA Y URBANISMO

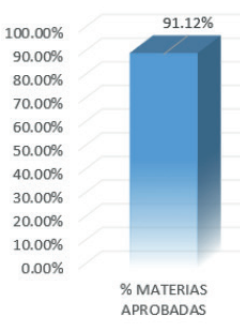

llustración 1: Elaboración Autores

\section{CIENCIAS ADMINISTRATIVAS}
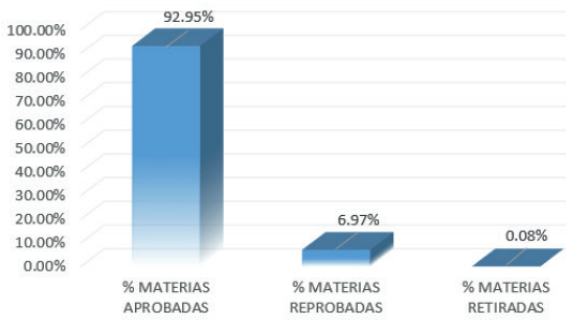

llustración 2: Elaboración Autores 


\section{CIENCIAS DE LA INGENIERÍA E} INDUSTRIAS

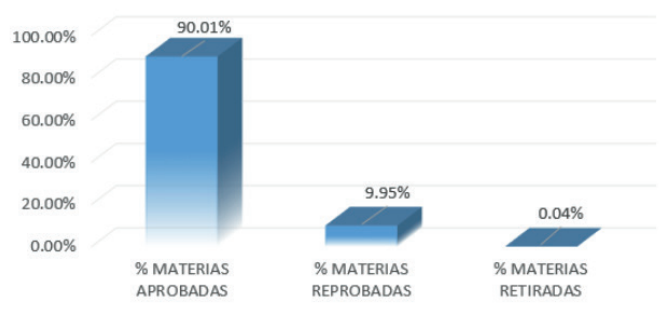

Ilustración 3: Elaboración Autores

\section{COMUNICACIÓN, ARTES Y HUMANIDADES}

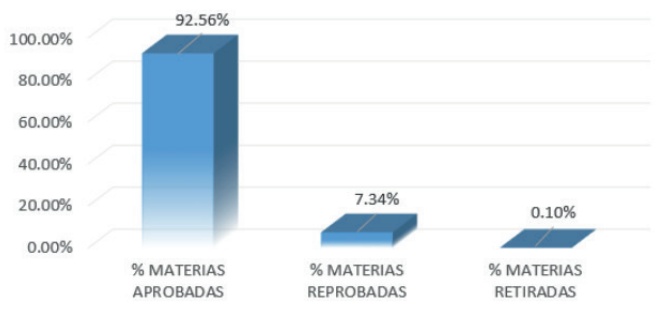

Ilustración 5: Elaboración Autores

La Facultad de Ciencias de la Ingeniería e Industrias es la que tiene el porcentaje más alto de materias reprobadas (9,95\%), esto se debe principalmente a que las asignaturas dictadas en los niveles inferiores son las que requieren en mayor proporción los conocimientos básicos para Física, Álgebra, Matemáticas y, Química; materias impartidas en el Bachillerato General Unificado (BGU). Torres (2018) dice que, en la Asamblea Nacional, se habla de la reforma a la Ley Orgánica de Educación Intercultural Bilingüe (LOEI), que implicaría la eliminación del BGU, que ofrece un grupo de asignaturas centrales denominado tronco común. Y se plantea volver al Bachillerato por Especialidades (BE), en el que los estudiantes debían escoger entre Químico-Biólogo, Físico-Matemático o Ciencias Sociales.

Por otro lado, a nivel de toda la Universidad se determina que del total de asignaturas registradas en primera matrícula, el 92,96\% se aprobaron, mientras que de los que toma-

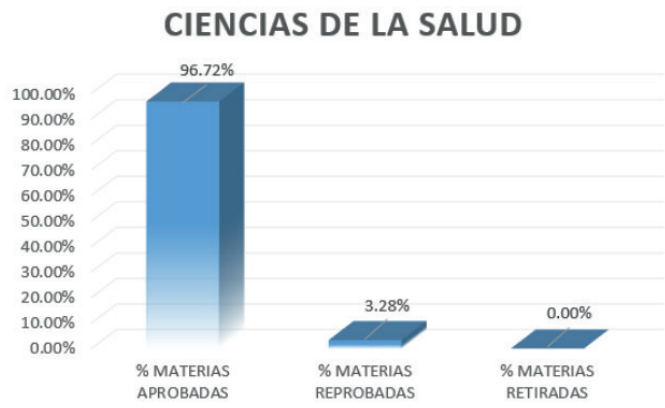

Ilustración 4: Elaboración Autores

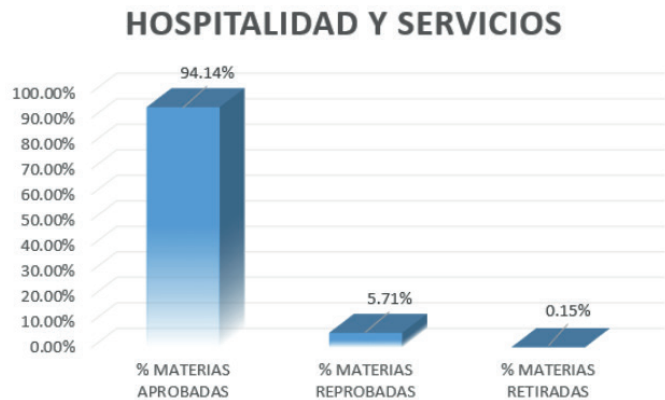

Ilustración 6: Elaboración Autores

ron materias con segunda matrícula, el porcentaje de aprobación disminuye al 87,75\%, y para los que toman materias en tercera matrícula, aprueban el 92,13\%; a nivel de facultad es Ciencias de la Ingeniería e Industrias la que tiene el porcentaje de aprobación más bajo es 90,28\% en primera matrícula (ver ilustración 7) este resultado se debe a las exigencias propias de la formación de carácter técnico y/o tecnológico de cada una de las asignaturas de la malla curricular. Mientras que el porcentaje más alto corresponde a Ciencias de la Salud ubicándose en el 96,79.

Referente a la segunda matrícula la facultad que menos inconvenientes tiene es Comunicación, Artes y Humanidades, $82,95 \%$, mientras que en las terceras matriculas está Facultad es la que menos aprueba, en cambio los alumnos de Ciencias de la Salud aprueban todos.

En la ilustración 7 se aprecian los porcentajes de aprobados por facultad y número de matrícula. 
Ilustración 7 Porcentajes de aprobados por facultad y número de matrícula

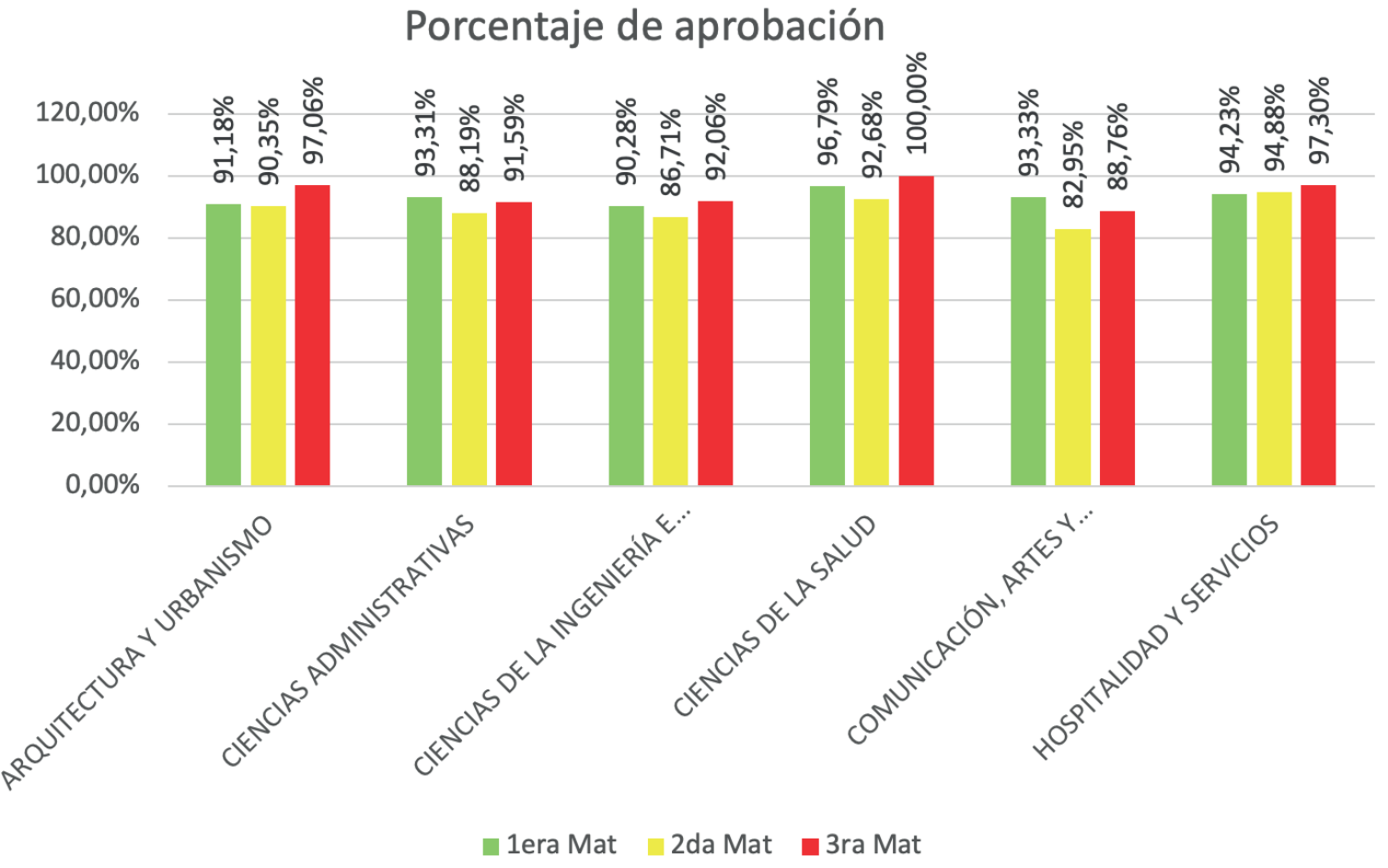

Elaboración Autores

\section{Periodos académicos para concluir el pensum de estudios}

Para determinar la cantidad de periodos académicos que se retrasaría un estudiante en promedio en la Universidad UTE Sede Quito, se utilizaron la cantidad de carreras y pensum de estudios mostrados en la tabla 5, así como la cantidad de estudiantes por nivel (tabla 6) de cada carrera y pensum y, el porcentaje de asignaturas (créditos) reprobadas (tabla 7) que se tiene en cada nivel del periodo abril 2017 / agosto 2017.

Tabla 5. Carreras por facultad

\begin{tabular}{|l|l|l|}
\hline \multicolumn{1}{|c|}{ Facultad } & \multicolumn{1}{c|}{$\begin{array}{c}\text { Cantidad } \\
\text { carreras }\end{array}$} & \multicolumn{1}{c|}{$\begin{array}{c}\text { Cantidad } \\
\text { pensum }\end{array}$} \\
\hline Arquitectura y urbanismo & 2 & 2 \\
\hline Ciencias Administrativas & 10 & 20 \\
\hline Ciencias de la Ingeniería e Industrias & 7 & 11 \\
\hline Ciencias de la Salud & 2 & 3 \\
\hline Comunicación, Artes y Humanidades & 15 & 21 \\
\hline Hospitalidad y Servicios & 7 & 13 \\
\hline Total & 43 & 70 \\
\hline
\end{tabular}


Tabla 6. Número de estudiantes por nivel

\begin{tabular}{|l|l|l|l|l|l|l|l|l|l|l|}
\hline \multirow{2}{*}{ Facultad } & \multicolumn{9}{|c|}{ Estudiantes por nivel } \\
\cline { 2 - 13 } & $\mathbf{1}$ & $\mathbf{2}$ & $\mathbf{3}$ & $\mathbf{4}$ & $\mathbf{5}$ & $\mathbf{6}$ & $\mathbf{7}$ & $\mathbf{8}$ & $\mathbf{9}$ & $\mathbf{1 0}$ \\
\hline Arquitectura y urbanismo & 105 & 105 & 115 & 98 & 63 & 46 & 61 & 56 & 65 & 0 \\
\hline Ciencias Administrativas & 348 & 299 & 304 & 338 & 312 & 301 & 279 & 282 & 263 & 7 \\
\hline Ciencias de la Ingeniería e Industrias & 391 & 305 & 301 & 295 & 256 & 248 & 213 & 240 & 257 & 0 \\
\hline Ciencias de la Salud & 190 & 118 & 113 & 96 & 98 & 81 & 68 & 104 & 15 & 55 \\
\hline Comunicación, Artes y Humanidades & 203 & 144 & 279 & 327 & 331 & 353 & 292 & 268 & 242 & 0 \\
\hline Hospitalidad y Servicios & 131 & 101 & 125 & 143 & 119 & 124 & 113 & 130 & 133 & 5 \\
\hline Total & 1368 & 1072 & 1237 & 1297 & 1179 & 1153 & 1026 & 1080 & 975 & 67 \\
\hline
\end{tabular}

Elaboración: Autores

Tabla 7. Porcentaje de reprobación por nivel

\begin{tabular}{|l|c|c|c|c|c|c|c|c|c|c|}
\hline \multirow{2}{*}{ Facultad } & \multicolumn{9}{|c|}{ Porcentaje de reprobación por nivel } \\
\cline { 2 - 11 } & $\mathbf{1}$ & $\mathbf{2}$ & $\mathbf{3}$ & $\mathbf{4}$ & $\mathbf{5}$ & $\mathbf{6}$ & $\mathbf{7}$ & $\mathbf{8}$ & $\mathbf{9}$ & $\mathbf{1 0}$ \\
\hline Arquitectura y urbanismo & $19.15 \%$ & $12.78 \%$ & $7.44 \%$ & $7.21 \%$ & $4.27 \%$ & $4.77 \%$ & $6.19 \%$ & $4.94 \%$ & $1.33 \%$ & \\
\hline Ciencias Administrativas & $21.17 \%$ & $10.49 \%$ & $9.05 \%$ & $6.82 \%$ & $5.39 \%$ & $4.55 \%$ & $3.35 \%$ & $2.53 \%$ & $3.62 \%$ & $0.00 \%$ \\
\hline $\begin{array}{l}\text { Ciencias de la Ingeniería e } \\
\text { Industrias }\end{array}$ & $23.35 \%$ & $20.88 \%$ & $10.34 \%$ & $8.85 \%$ & $8.87 \%$ & $6.03 \%$ & $4.75 \%$ & $3.13 \%$ & $1.58 \%$ & \\
\hline Ciencias de la Salud & $3.00 \%$ & $7.29 \%$ & $3.81 \%$ & $6.03 \%$ & $1.64 \%$ & $3.44 \%$ & $1.84 \%$ & $0.24 \%$ & $0.00 \%$ & $0.76 \%$ \\
\hline $\begin{array}{l}\text { Comunicación, Artes y } \\
\text { Humanidades }\end{array}$ & $15.04 \%$ & $15.10 \%$ & $14.05 \%$ & $9.92 \%$ & $7.37 \%$ & $7.96 \%$ & $4.73 \%$ & $4.72 \%$ & $3.89 \%$ & \\
\hline Hospitalidad y Servicios & $11.27 \%$ & $10.81 \%$ & $8.14 \%$ & $5.76 \%$ & $5.16 \%$ & $5.02 \%$ & $4.14 \%$ & $3.62 \%$ & $3.07 \%$ & $0.00 \%$ \\
\hline Total & $17.27 \%$ & $13.96 \%$ & $9.77 \%$ & $7.92 \%$ & $6.31 \%$ & $5.90 \%$ & $4.19 \%$ & $3.24 \%$ & $2.87 \%$ & $0.62 \%$ \\
\hline
\end{tabular}

Elaboración: Autores

En la tabla 8 se consideran las carreras y modalidad, se excluyen para el análisis las carreras que tienen estudiantes únicamente en los dos últimos niveles.

Tabla 8. Tipo de modalidad

\begin{tabular}{|l|l|l|}
\hline \multicolumn{1}{|c|}{ FACULTAD } & \multicolumn{1}{c|}{ CARRERA } & MODALIDAD \\
\hline Arquitectura Y Urbanismo & Arquitectura & Presencial \\
\hline Ciencias Administrativas & Arquitectura Interior & Presencial \\
\hline Ciencias Administrativas & Administración De Empresas De Servicio Y Recursos Humanos & Distancia \\
\hline Ciencias Administrativas & Ingeniería De Empresas Y Negocios & Presencial \\
\hline Ciencias Administrativas & Ingeniería En Administración Del Talento Humano & Presencial \\
\hline Ciencias Administrativas & Ingeniería En Comercio Exterior, Integración Y Aduanas & Presencial \\
\hline Ciencias Administrativas & Ingeniería En Contabilidad Y Auditoría Cpa & Presencial \\
\hline Ciencias Administrativas & Ingeniería En Finanzas Y Auditoria Cpa & Presencial \\
\hline Ciencias Administrativas & Ingeniería En Marketing & Presencial \\
\hline Ciencias De La Ingeniería E Industrias & Ingeniería Ambiental Y Manejo De Riesgos Naturales & Presencial \\
\hline Ciencias De La Ingeniería E Industrias & Ingeniería Automotriz & \\
\hline
\end{tabular}




\begin{tabular}{|l|l|l|}
\hline Ciencias De La Ingeniería E Industrias & Ingeniería De Alimentos & Presencial \\
\hline Ciencias De La Ingeniería E Industrias & Ingeniería De Petróleos & Presencial \\
\hline Ciencias De La Ingeniería E Industrias & Ingeniería Industrial Y De Procesos & Presencial \\
\hline Ciencias De La Ingeniería E Industrias & Ingeniería Informática Y Ciencias De La Computación & Presencial \\
\hline Ciencias De La Ingeniería E Industrias & Ingeniería Mecatrónica & Presencial \\
\hline Ciencias De La Salud & Medicina & Presencial \\
\hline Ciencias De La Salud & Odontología & Presencial \\
\hline Comunicación, Artes Y Humanidades & Ciencias De La Educación Mención Educación Básica & Distancia \\
\hline Comunicación, Artes Y Humanidades & Ciencias De La Educación Mención Lenguaje Y Comunicación & Distancia \\
\hline Comunicación, Artes Y Humanidades & Ciencias De La Educación Mención Matemáticas & Distancia \\
\hline Comunicación, Artes Y Humanidades & Diseño De Modas & Presencial \\
\hline Comunicación, Artes Y Humanidades & Diseño Gráfico Publicitario & Presencial \\
\hline Comunicación, Artes Y Humanidades & Educación Inicial & Semipresencial \\
\hline Comunicación, Artes Y Humanidades & Periodismo & Presencial \\
\hline Comunicación, Artes Y Humanidades & Publicidad Y Gestión & Presencial \\
\hline Comunicación, Artes Y Humanidades & Relaciones Públicas Y Comunicación Organizacional & Presencial \\
\hline Hospitalidad Y Servicios & Administración De Empresas Turísticas Y Conservación Ambiental & Distancia \\
\hline Hospitalidad Y Servicios & Presencial \\
\hline Hospitalidad Y Servicios & Administración Hotelera & Presencial \\
\hline
\end{tabular}

Fuente: SICAF

Con el porcentaje de asignaturas reprobadas por nivel (tabla 9) y con el número promedio de asignaturas por nivel de cada pensum, se obtuvo el número de periodos (niveles) adicionales que un estudiante se requeriría para completar el pensum de estu- dios. No se consideraron los retiros y reingresos por tener porcentajes no significativos, de igual manera en la Facultad de Ciencias de la Salud no se tomaron en cuenta los dos periodos del internado rotativo.

Tabla 9. Porcentaje de reprobación por nivel

\begin{tabular}{|c|c|c|c|c|c|c|c|c|c|c|}
\hline \multirow{2}{*}{ Carrera } & \multicolumn{10}{|c|}{ Porcentaje de reprobación por nivel } \\
\hline & 1 & 2 & 3 & 4 & 5 & 6 & 7 & 8 & 9 & 10 \\
\hline Arquitectura & $18.55 \%$ & $11.70 \%$ & $8.39 \%$ & $8.71 \%$ & $4.69 \%$ & $5.48 \%$ & $6.93 \%$ & $5.27 \%$ & $2.06 \%$ & \\
\hline Arquitectura Interior & $50.00 \%$ & $18.35 \%$ & $3.41 \%$ & $2.33 \%$ & $2.27 \%$ & $1.85 \%$ & $4.56 \%$ & $3.85 \%$ & $0.00 \%$ & \\
\hline $\begin{array}{l}\text { Administración De Empresas De Servicio } \\
\text { Y Recursos Humanos }\end{array}$ & $28.97 \%$ & $12.14 \%$ & $11.18 \%$ & $10.00 \%$ & $4.60 \%$ & $10.66 \%$ & $4.49 \%$ & $1.19 \%$ & $7.91 \%$ & \\
\hline Ingeniería De Empresas Y Negocios & $29.40 \%$ & $12.50 \%$ & $3.82 \%$ & $10.55 \%$ & $4.17 \%$ & $2.06 \%$ & $5.03 \%$ & $0.00 \%$ & $0.81 \%$ & \\
\hline $\begin{array}{l}\text { Ingeniería En Administración Del Talento } \\
\text { Humano }\end{array}$ & $12.85 \%$ & $9.46 \%$ & $8.04 \%$ & $2.09 \%$ & $4.44 \%$ & $0.90 \%$ & $1.96 \%$ & $5.00 \%$ & $2.25 \%$ & \\
\hline $\begin{array}{l}\text { Ingeniería En Comercio Exterior, Integra- } \\
\text { ción Y Aduanas }\end{array}$ & $11.94 \%$ & $10.09 \%$ & $9.19 \%$ & $8.05 \%$ & $6.14 \%$ & $2.51 \%$ & $2.80 \%$ & $1.09 \%$ & $5.14 \%$ & \\
\hline $\begin{array}{l}\text { Ingeniería En Contabilidad Y Auditoría } \\
\text { Cpa }\end{array}$ & $34.45 \%$ & $15.80 \%$ & $12.08 \%$ & $4.13 \%$ & $8.28 \%$ & $5.19 \%$ & $5.68 \%$ & $10.00 \%$ & $2.94 \%$ & \\
\hline Ingeniería En Finanzas Y Auditoria Cpa & $17.77 \%$ & $5.33 \%$ & $3.75 \%$ & $8.05 \%$ & $6.29 \%$ & $1.70 \%$ & $1.48 \%$ & $2.80 \%$ & $1.72 \%$ & \\
\hline Ingeniería En Marketing & $18.82 \%$ & $8.77 \%$ & $14.38 \%$ & $2.95 \%$ & $1.94 \%$ & $10.05 \%$ & $4.09 \%$ & $4.39 \%$ & $0.00 \%$ & \\
\hline
\end{tabular}




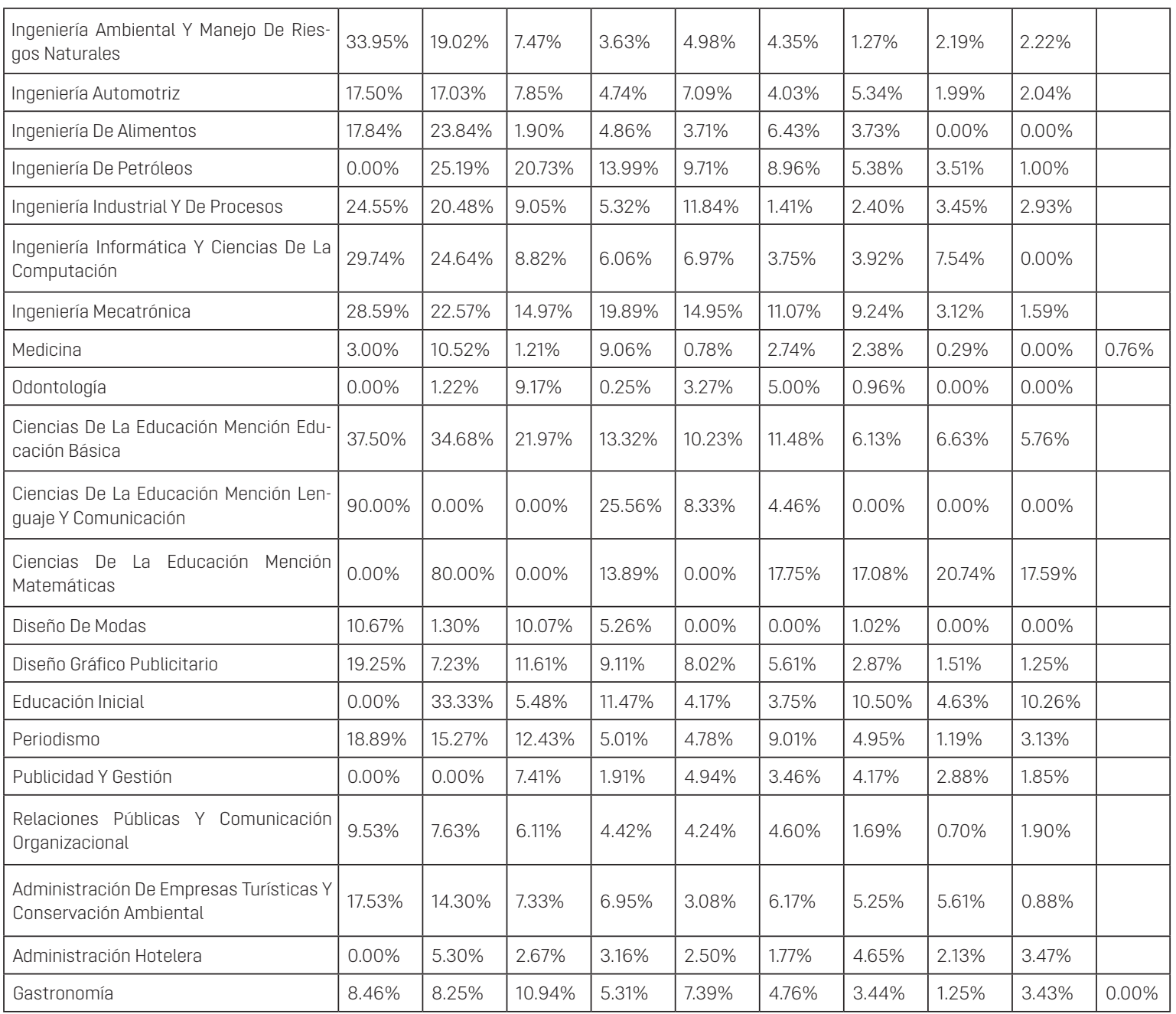

Elaboración: Autores

La tabla 10 evidencia el tiempo o número de periodos (niveles) adicionales que un estudiante en promedio requerirá para aprobar la malla curricular por facultad.

Tabla 10. Periodos adicionales para aprobar la malla curricular por facultad

\begin{tabular}{|l|l|l|}
\hline \multicolumn{1}{|c|}{ Facultad } & \multicolumn{1}{|c|}{ FACULTAD } & \multicolumn{1}{|c|}{$\begin{array}{c}\text { Cantidad máxi- } \\
\text { ma de periodos } \\
\text { adicionales }\end{array}$} \\
\hline Arquitectura y urbanismo & ARQUITECTURA Y URBANISMO & 1 \\
\hline Ciencias Administrativas & CIENCIAS ADMINISTRATIVAS & 1 \\
\hline Ciencias de la Ingeniería e Industrias & CIENCIAS DE LA INGENIERÍA E INDUSTRIAS & 2 \\
\hline Ciencias de la Salud & CIENCIAS DE LA SALUD & 1 \\
\hline Comunicación, Artes y Humanidades & COMUNICACIÓN, ARTES Y HUMANIDADES & 2 \\
\hline Hospitalidad y Servicios & HOSPITALIDAD Y SERVICIOS & 1 \\
\hline
\end{tabular}


En los resultados de la tabla anterior se incluyen todas las asignaturas obligatorias y optativas de la malla curricular, es decir, materias básicas, materias de profesionalización, optativas e idiomas extranjeros.

El idioma extranjero en la gran mayoría de carreras de la Universidad UTE sede Quito, ha sido uno de los más importantes impedimentos para que los estudiantes concluyan la malla curricular (pensum) dentro del tiempo ideal, pero en este caso no se debe a la repetición, sino a que los estudiantes no se matriculaban en esta, por consiguiente, retrasaban su aprobación.

El rendimiento académico en los tres primeros niveles no es satisfactorio, debido a que la repitencia en éstos son los que más retrasan el tiempo de finalización del pensum de estudios (Tabla 11), de tal forma que se determinó que los estudiantes requieren de hasta dos niveles más para culminar el pensum de estudios. Cabe señalar que este fenómeno exige a la Universidad UTE implementar continuamente nuevas metodologías de aprendizaje para igualar los vacíos que deja el BGU, para lo cual el Sistema de Educación Superior debe tener suficiente información con el fin, como manifiesta González, (2011), de generar métodos que faciliten el conocimiento de las relaciones y complejidades de lo que aprendemos para poder contextualizar en algo más global que cobre sentido.

Tabla 11. Repitencia en los 5 primeros niveles

\begin{tabular}{|l|l|l|l|l|l|}
\hline \multirow{2}{*}{ Facultad } & \multicolumn{5}{c|}{ Repitencia en los 5 primeros niveles } \\
\cline { 2 - 6 } & \multicolumn{1}{c|}{$\mathbf{1}$} & $\mathbf{2}$ & $\mathbf{3}$ & $\mathbf{4}$ & \multicolumn{1}{c|}{$\mathbf{5}$} \\
\hline Arquitectura y urbanismo & $19.36 \%$ & $11.77 \%$ & $7.69 \%$ & $7.36 \%$ & $4.27 \%$ \\
\hline Ciencias Administrativas & $23.27 \%$ & $10.86 \%$ & $9.62 \%$ & $6.84 \%$ & $5.54 \%$ \\
\hline Ciencias de la Ingeniería e Industrias & $23.32 \%$ & $19.81 \%$ & $10.34 \%$ & $8.76 \%$ & $8.76 \%$ \\
\hline Ciencias de la Salud & $3.00 \%$ & $7.21 \%$ & $3.81 \%$ & $8.12 \%$ & $2.78 \%$ \\
\hline Comunicación, Artes y Humanidades & $15.03 \%$ & $15.34 \%$ & $14.37 \%$ & $9.97 \%$ & $7.34 \%$ \\
\hline Hospitalidad y Servicios & $11.30 \%$ & $11.33 \%$ & $8.54 \%$ & $5.70 \%$ & $5.42 \%$ \\
\hline
\end{tabular}

Elaboración: Autores

\section{Conclusiones}

Todas las carreras en promedio requieren al menos un periodo adicional para completar el pensum de estudios, pero para Ciencias de la Ingeniería e Industrias y para Comunicación, Artes y Humanidades la cantidad de niveles llega hasta dos, en esta última, son las carreras de modalidad a distancia las que un mayor número de periodos necesitan.

En todas las facultades, excepto en Ciencias de la Salud, se puede determinar que en los tres primeros niveles de las carreras se tiene el porcentaje de reprobación más alto y partir del cuarto este empieza a decrecer, lo que implica que el rendimiento académico de los estudiantes mejora.

De estos resultados, se puede establecer que el incremento de periodos académicos para culminar la malla curricular se relaciona con la repitencia asociada a la seriación de asignaturas (prerrequisitos y correquisitos) en las trayectorias individuales de cada uno de los estudiantes.

Se debería eliminar el BGU, que ofrece un grupo de asignaturas centrales denominado tronco común, para volver al $\mathrm{BE}$, en el que los estudiantes debían escoger entre Químico-Biólogo, Físico-Matemático o Ciencias Sociales, con esto lograríamos reducir 
sustancialmente la repitencia en los primeros niveles de la Universidad.

En el estudio realizado se considera el efecto de la repitencia en el tiempo de culminación de la malla curricular y no el efecto que tiene el no cursar una asignatura o lo que es lo mismo retrasar su aprobación, como es el caso del idioma extranjero.

\section{Referencias}

Álvarez, M., Gómez, E., \& Morfín, M. (2012). Efecto de la beca CONACYTen la eficiencia terminal en el posgrado. Revista Electrónica de Investigación Educativa, 14. Retrieved from http://www.scielo.org.mx/pdf/redie/ v14n1/v14n1a10.pdf

Esparza, D., \& López, R. (2011). Perfil de ingreso de alumnos con buen desempeño académico en el primer año de estudios: El caso de la Escuela de Diseño de la Universidad De La Salle Bajío. Revista de Investigación de La Universidad de La Salle Bajío, 3. Retrieved from http://www.scielo.org.mx/pdf/ns/ v3n6/v3n6a6.pdf

Guadalupe, C. (2005). El impacto de la escuela de procedencia del nivel medio superior en el desempeño de los alumnos en el nivel universitario. Revista Electrónica Iberoamericana Sobre Calidad, Eficacia y Cambio En Educación, 3.

López, A., Albíter, Á., \& Ramírez, L. (2008). Eficiencia terminal en la educación superior, la necesidad de un nuevo paradigma. Revista de La Educación Superior, 37. Retrieved from http://www.scielo.org.mx/pdf/resu/ v37n146/v37n146a9.pdf

Torres, M.(2018) Especialidades o Unificado, los bachilleratos tienen defensores, recuperado de https://www.expreso.ec/guayaquil/bachillerato-especialidades-unificado-leyorganicadeeducacion-asambleaJD2511821

Passailaigue, R., Amechazurra, 0., \& Galarza, J. (2013). La deserción y la repitencia en las instituciones de educación superior: algunas experiencias investigativas en el Ecuad. Revista Cientifica de La Universidad de Cienfuegos, 6.

Vera, A., Ramos, Y., Sotelo, A., Echeverría, S., \& Serrano, M. (2012). Factores asociados al rezago en estudiantes de una institución de educación superior en México. Revista Iberoamericana de Educación Superior, 3. Retrieved from http://www.scielo.org.mx/ pdf/ries/v3n7/v3n7a3.pdf

Guavara, G. et al, (2016), La evolución Docente en el Mundo, Instituto Nacional para la la Evaluación de la Educación, Mexico, p. 195

Cavagnaro, J. (2019), ¿Qué estudian los ecuatorianos? Revista Vistazo, No. 1252,octubre 25, p.64

González, A. (2011) Los 7 saberes para la educación del futuro. Edgar Morin, recuperado de http://cisolog.com/sociologia/los-7-sabe res-para-la-educacion-del-futuro-edgarmorin/ 